TRANSACTIONS OF THE

AMERICAN MATHEMATICAL SOCIETY

Volume 361, Number 10, October 2009, Pages 5331-5343

S 0002-9947(09)04646-7

Article electronically published on May 28, 2009

\title{
A CLASS OF FINITE SIMPLE BOL LOOPS OF EXPONENT 2
}

\author{
GÁBOR P. NAGY
}

\begin{abstract}
In this paper we give an infinite class of finite simple right Bol loops of exponent 2. The right multiplication group of these loops is an extension of an elementary Abelian 2-group by $S_{5}$. The construction uses the description of the structure of such loops given by M. Aschbacher (2005). These results answer some questions of M. Aschbacher.
\end{abstract}

\section{INTRODUCTION}

The set $Q$ endowed with a binary operation $x \cdot y=x y$ is a loop if it has a unit element 1 and the equation $x y=z$ has a unique solution whenever two of the three unknowns are given. The loop $Q$ is a (right) Bol loop if the identity

$$
((x y) z) y=x(y(z y))
$$

holds for all $x, y, z \in Q$. Bol loops of exponent 2 play an important role in the theory of loops and are related to interesting group theoretical problems. Using the socalled Baer correspondence, this class of loops can be described by a triple $(G, H, K)$ with group $G$, core-free subgroup $H \leq G$ and system of coset representatives $K \subseteq G$ such that $1 \in K, G=\langle K\rangle$ and $K \backslash\{1\}$ is a union of conjugacy classes of involutions.

Bol loops of exponent 2 which are not elementary Abelian groups have long been known to exist; the first construction is due to R. P. Burn [7. Later, many infinite classes of such loops were given; see [11, 12, 13, 16. All of these examples were solvable loops; equivalently the group $G$ was a 2-group. The existence of nonsolvable finite Bol loops of exponent 2 was considered as one of the main open problem in the theory of loops and quasigroups. As the smallest such loop must be simple, this question was related to the existence of finite simple proper right Bol loops. Here by proper we mean right Bol loops which are not Moufang, that is, which do not satisfy the identity $x(y x)=(x y) x$. Finite simple proper Bol loops were constructed by the author [17] recently.

By [15, the solvability of a Bol loop of 2-power exponent is equivalent to having 2power order. Later, S. Heiss $[9$ showed that the solvability of the loop corresponding to the triple $(G, H, K)$ is equivalent to the solvability of the group $G$. The next major step was the paper [3] by M. Aschbacher. His main result gives a detailed description on the structure of the right multiplication group of minimal nonsolvable Bol loops of exponent 2. This result was achieved by using the classification of finite simple groups.

Received by the editors July 25, 2007 and, in revised form, September 19, 2007.

2000 Mathematics Subject Classification. Primary 20N05; Secondary 20C20, 20 F29.

This paper was written during the author's Marie Curie Fellowship MEIF-CT-2006-041105.

(C)2009 American Mathematical Society

Reverts to public domain 28 years from publication 
In this paper we apply Aschbacher's recipe to construct a class of finite simple Bol loops of exponent 2. In this way, we give a negative answer to questions 2 and 3 of $[3$ and [4. The smallest member of our class has order 96. We emphasize that this example is small and the structural description of the smallest example in [3] and [4 is so precise that it was only a matter of time until somebody found it by some short computer calculation. This explains the fact that this loop was indepently discovered by the author and by B. Baumeister and A. Stein [5] with a time delay of 10 days.

\section{BASIC CONCEPTS}

In order to make this paper self-contained, we define the basic loop theoretical concepts. Subloops, homomorphisms and normal subloops can be defined for the class of loops similarly to groups; see [6]. The loop $Q$ is said to be simple if it has no proper normal subloops.

For a loop $Q$, the maps $L_{a}, R_{a}: Q \rightarrow Q, L_{a}(x)=a x, R_{a}(x)=x a$ are the left and right multiplication maps of $Q$. They are bijections of $Q$ and generate the left and right multiplication groups of $Q$, respectively. The section $S(Q)$ of the loop $Q$ is the set of the right multiplication maps of $Q$.

The right Bol identity can equivalently be expressed by $R_{x} R_{y} R_{x} \in S(Q)$ for all $x, y \in Q$. Bol loops are power-associative; that is, $x^{n}$ is well defined for all $x \in Q$ and $n \in \mathbb{Z}$. The order of the element $x$ is the smallest positive integer $n$ such that $x^{n}=1$. The exponent of $Q$ is the smallest positive integer $n$ such that $x^{n}=1$ for each $x \in Q$.

We will formulate our results by using the Baer correspondence between the class of loops and the class of triples $(G, H, K)$ where $G$ is a group, $H \leq G$ and $K$ is a set of coset representatives of all conjugates $H^{g}$ in $G$, and $1 \in K$. For a given loop $Q$, $G$ can be chosen to be the right multiplication group, $H$ the stabilizer of the unit element and $K$ the set of right multiplication maps. Conversely, for a given triple $(G, H, K)$, a loop can be constructed in the following way. We define the operation $x \circ y$ on $K$ by $H(x \circ y)=H x y$; then $Q=(K, \circ)$ is a loop. The triple satisfying the above condition will be said to be a loop folder.

The loop folder $(G, H, K)$ determines a Bol loop of exponent 2 if and only if $K=\{1\} \cup \bigcup_{i \in I} C_{i}$, where the $C_{i}$ 's are conjugacy classes of involutions in $G$.

\section{The FIRST CONSTRUCTION}

As usual, $S_{5}$ and $P G L(2,5)$ are the permutation groups acting on 5 and 6 points, respectively. It is well known that $S_{5} \cong P G L(2,5) \cong \operatorname{Aut}\left(L_{2}(4)\right) \cong O_{4}^{-}(2)$, where $\operatorname{Aut}\left(L_{2}(4)\right)$ is the extension of $L_{2}(4)=P S L(2,4) \cong A_{5}$ by a field automorphism of order 2, and $O_{4}^{-}(2)$ is the orthogonal group on a 4-dimensional orthogonal space over $\mathbb{F}_{2}$ of Witt index 1 . We denote by $F_{20}$ the affine linear group acting on $\mathbb{F}_{5}$, $F_{20} \cong C_{5} \rtimes C_{4}$. On the one hand, $F_{20}$ is the Borel subgroup of $\operatorname{PGL}(2,5)$, that is, the stabilizer of a projective point. On the other hand, $F_{20} \leq S_{5}$ is a sharply 2 -transitive permutation group on 5 points.

In the sequel, we define a group $G$ which is a nonsplit extension of the elementary Abelian group of order 32 by $S_{5}$ such that the transpositions of $S_{5}$ lift to involutions in $G$ and the even involutions of $S_{5}$ lift to elements of order 4 . Despite the relatively small order of $G$, we found no simple description for this group; therefore our definion will be rather ad hoc, as well. We start with two technical lemmas. 
Lemma 3.1. We have the following presentations of groups with generators and relations.

$$
\begin{aligned}
A_{5} & =\left\langle a, b \mid a^{2}=b^{3}=(a b)^{5}=1\right\rangle, \\
S_{5} & =\left\langle c, d \mid c^{2}=d^{4}=(c d)^{5}=[c, d]^{3}=1\right\rangle, \\
2 . S_{5} & =\left\langle C, D \mid C^{2}=D^{8}=(C D)^{5}=[C, D]^{3}=\left[C, D^{4}\right]=1\right\rangle,
\end{aligned}
$$

where $2 . S_{5}$ denotes the nonsplit central extension of $S_{5}$ in which the transpositions lift to involutions. In other words, $2 . S_{5}$ is the semidirect product of $S L(2,5)=2 . A_{5}$ with a group of order 2 .

Proof. The presentation for $A_{5}$ is well known. Assume $G=\langle c, d\rangle$ is the group presented by the second set of relations above. We observe first that $S_{5}$ satisfies these relations; hence no relation can collapse. Put $a=d^{2}$ and $b=[d, c]=[c, d]^{-1}$. Then $a^{2}=b^{3}=1$ and $a b=(d c)^{2}=\left((c d)^{2}\right)^{c}$. This latter implies $(a b)^{5}=1$; hence $G_{0}=\langle a, b\rangle \cong A_{5}$. Moreover, since $d c=(d c)^{6}=(a b)^{3} \in G_{0}$, we have $c d=d c[c, d]=d c b^{-1} \in G_{0}$ and

$$
d b d^{-1}=c d c d^{-1}=(c d)^{2} d^{-2} \in G_{0} .
$$

This means that $d$ and $c d$ normalize $G_{0}$, so $G_{0} \triangleleft G$. So $\left|G: G_{0}\right|=2$ and $d \notin G_{0}$ since $A_{5}$ contains no element of order 4 . This proves $G \cong S_{5}$. Finally, $D^{4}$ is a central involution in $H=\langle C, D\rangle$ and $H /\left\langle D^{4}\right\rangle$ maps surjectively to $S_{5}$. The order of $D$ is 8 ; thus, the extension is nonsplit and the involution $C$ covers a transposition in $S_{5}$.

Lemma 3.2. The permutations

$$
\begin{aligned}
c= & (1,4)(2,9)(3,10)(6,11)(7,12)(13,21)(14,22)(15,24)(16,23)(17,30) \\
& (18,29)(19,31)(20,32)(33,35)(38,40) \\
d= & (1,2,4,6,8,7,5,3)(9,13,25,18,10,14,26,17)(11,15,27,20,12,16, \\
& 28,19)(21,30,38,34,23,31,40,35)(22,32,39,36,24,29,37,33)
\end{aligned}
$$

acting on 40 points satisfy the relations

$$
c^{2}=d^{8}=(c d)^{5}=[c, d]^{3}=\left[d^{4}, c\right]^{2}=\left[d^{4}, c d c d^{-2} c\right]=1 .
$$

Moreover, with $u_{1}=d^{4}, u_{2}=u_{1}^{c}, u_{3}=u_{1}^{c d}, u_{4}=u_{1}^{c d c}, u_{5}=u_{1}^{c d c d}, u_{6}=u_{1}^{c d c d c}$ the identity $u_{1} u_{2} u_{3} u_{4} u_{5} u_{6}=1$ holds.

Proof. We leave the straightforward calculations to the reader.

Lemma 3.3. The group $G=\langle c, d\rangle$ given in Lemma 3.2 satisfies

$G$ has an elementary Abelian normal subgroup $J$ of order 32 such that

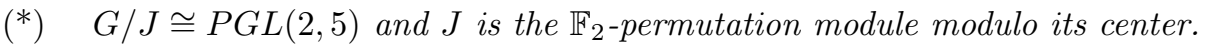
Moreover, $[G, G] /[G, J] \cong S L(2,5)$ and $G$ splits over $[G, G] J$.

Proof. We claim that the conjugacy class of $u_{1}=d^{4}$ in $G$ is $X=\left\{u_{1}, \ldots, u_{6}\right\}$. It is immediate that $c$ induces the permutation $\left(u_{1} u_{2}\right)\left(u_{3} u_{4}\right)\left(u_{5} u_{6}\right)$ on $X$. Moreover, $d$ centralizes $u_{1}$ and maps $u_{2} \mapsto u_{3}, u_{4} \mapsto u_{5}$. From the last relation in (1), it follows that $u_{1}^{c d c}=u_{1}^{c d^{2}}$; hence $u_{3}^{d}=u_{3}^{c}=u_{4}$. By $\left[d^{4}, c\right]^{2}=1$ we have

$$
u_{1}^{c d^{4} c}=c d^{4} c d^{4} c d^{4} c=d^{4}\left[d^{4}, c\right]^{2}=d^{4}=u_{1}
$$


thus $u_{2}=u_{1}^{c}=u_{1}^{c d^{4}}=u_{4}^{d^{2}}=u_{5}^{d}$. To see that $d$ acts on $X$, we need to show that $d$ centralizes $u_{6}$ :

$$
u_{6}^{d}=u_{1}^{c d c d c d}=u_{1}^{(c d)^{-2}}=u_{1}^{d^{-1} c d^{-1} c}=u_{1}^{c d^{-1} c}=u_{2}^{d^{-1} c}=u_{5}^{c}=u_{6} .
$$

The action of $d$ on $X$ is therefore $\left(u_{2} u_{3} u_{4} u_{5}\right)$. This not only shows that $X$ is a conjugacy class in $G$, but we also have the action of $G$ on $X$. Indeed, one shows by straightforward calculation that $\tilde{c}=(12)(34)(56)$ and $\tilde{d}=(2345)$ satisfy the relations of $S_{5}$ from Lemma 3.1. Since the action of $S_{5}$ on 6 points is unique, we have $G / C_{G}(X) \cong P G L(2,5)$.

As $\left[u_{1}, u_{2}\right]=\left[d^{4}, c d^{4} c\right]=\left[d^{4}, c\right]^{2}=1$ and $P G L(2,5)$ acts 2-transitively, $\left[u_{i}, u_{j}\right]=$ 1 holds for all $i, j$. This means that $J=\langle X\rangle$ is an elementary Abelian 2-group and $|J|=32$ by $u_{1} \cdots u_{6}=1$. Using the presentation of $2 . S_{5}$ from Lemma 3.1. $G / J_{0} \cong 2 . S_{5}$. This implies

$$
[G, G] /[G, J] \cong\left[G / J_{0}, G / J_{0}\right] \cong 2 \cdot A_{5} \cong S L(2,5) .
$$

Finally, $G$ splits over $[G, G] J$ as $c \notin[G, G] J$.

In the sequel, $G$ will denote a group satisfying $\left(^{*}\right)$. We would like to make clear that it can be shown using the computer algebra system GAP [8] that the group given in Lemma 3.2 is the unique group with this property. However, we hope that this more general approach will help in future generalization of the constructions of this paper.

Among other properties, we show in the next lemma that for our group $G$, $G^{\prime}=[G, G]$ is a perfect group. Actually, we found $G^{\prime}$ by using the library of perfect groups in the computer algebra system GAP [8] and constructed $G$ as a split extension of $G^{\prime}$ by an outer automorphism of order 2 .

Lemma 3.4. Let $G$ be a group satisfying $(*)$ and define $J_{0}=[G, J]$.

(i) We have $G^{\prime \prime}=G^{\prime}=[G, G]=[G, G] J$ and $\left|G: G^{\prime}\right|=2$.

(ii) $G \backslash J$ contains a unique class $c^{G}$ of involutions, and $\left|c^{G}\right|=80$. In particular, all involutions of $G^{\prime}=[G, G]=[G, G] J$ lie in $J$.

(iii) Let $P$ be a Sylow 5-subgroup. Then $N_{J_{0}}(P)=\{1\}$ and $N_{G}(P) \cong C_{8} \ltimes C_{5}$. Moreover, if the subgroup $U \leq G$ maps onto $F_{20}$ modulo $J$, then $U=N_{G}(P)$ or $U=N_{G}(P) J_{0}$.

Proof. (i) Let $V$ be the permutation $\mathbb{F}_{2}$-module of $P G L(2,5)$ with basis $\left\{u_{1}, \ldots, u_{6}\right\}$. Due to the 2-transitivity, the orbit of the element $u_{1}+u_{2}$ consists of the elements $u_{i}+u_{j}, i \neq j$, which are different modulo the center $\left\langle u_{1}+\ldots+u_{6}\right\rangle$ of $V$. Hence both $P G L(2,5)$ and $P S L(2,5)$ act transitively on the nonidentity elements of $J_{0}=[G, J]$, which implies that $J_{0}$ is a minimal normal subgroup in $G$ and $[G, G] J$. It follows that $J_{0}=\left[G^{\prime}, J_{0}\right]$ and $G^{\prime \prime} / J_{0}=\left(G^{\prime} / J_{0}\right)^{\prime}=G^{\prime} / J_{0}$ by $S L(2,5)^{\prime}=S L(2,5)$. This means $G^{\prime \prime}=G^{\prime}$. Finally, $J \leq G^{\prime}$ follows from $J / J_{0}=Z\left(G / J_{0}\right) \leq\left(G / J_{0}\right)^{\prime}=G^{\prime} / J_{0}$.

(ii) Since $G$ splits over $G^{\prime}=G^{\prime} J$, we can take an involution $c$ from $G \backslash G^{\prime}$; the image of $c$ in $G / J \cong S_{5}$ is a transposition. As $J$ is the permutation module modulo its center, $\operatorname{dim}_{\mathbb{F}_{2}}\left(C_{J_{0}}(c)\right)=2$ and $\operatorname{dim}_{\mathbb{F}_{2}}\left(C_{J}(c)\right)=3$. It is easy to check that $2 . S_{5} \cong G / J_{0}$ contains 20 noncentral involutions and they are all conjugate.

Let $c^{\prime}$ be another involution in $G \backslash J$; we want to show that $c, c^{\prime}$ are conjugate. For some $g \in G,\left(c J_{0}\right)^{g J_{0}}=c^{\prime} J_{0}$; that is, $c^{g} \in c^{\prime} J_{0}$. Hence we can assume $c \in c^{\prime} J_{0}$, $c^{\prime}=c j$ with $j \in J_{0}$. The element $c j$ has order 2 if and only if $j \in C_{J_{0}}(c)$. On the 
one hand, $c^{J_{0}} \subseteq c C_{J_{0}}(c)$. On the other hand,

$$
\left|c^{J_{0}}\right|=\left|J_{0}: C_{J_{0}}(c)\right|=4=\left|C_{J_{0}}(c)\right|=\left|c C_{J_{0}}(c)\right| .
$$

This implies $c^{J_{0}}=c C_{J_{0}}(c)$ and $c^{\prime} \in c^{J_{0}}$. No involution of $G^{\prime}$ can be conjugate to $c$; hence all involutions of $G^{\prime}$ must lie in $J$. Finally, we show $\left|c^{G}\right|=80$. As $c^{g} \in c J_{0}$ if and only if $c^{g}=c^{j}$ for some $j \in J_{0}$, we have $N_{G}\left(c J_{0}\right)=C_{G}(c) J_{0}$. Moreover, $C_{G / J_{0}}\left(c J_{0}\right)=N_{G}\left(c J_{0}\right) / J_{0}$. Thus,

$$
\begin{aligned}
\left|G: C_{G}(c)\right| & =\left|G: N_{G}\left(c J_{0}\right)\right|\left|C_{G}(c) J_{0}: C_{G}(c)\right| \\
& =\left|G / J_{0}: C_{G / J_{0}}\left(c J_{0}\right)\right|\left|J_{0}: C_{J_{0}}(c)\right| \\
& =\left|\left(c J_{0}\right)^{G / J_{0}}\right|\left|c^{J_{0}}\right|=20 \cdot 4=80 .
\end{aligned}
$$

(iii) $P$ acts fixed point free on the involutions of $J_{0}$; thus, $N_{J_{0}}(P)=1$. Moreover, $5 \nmid|J|-1$; hence $P$ centralizes a unique element $a \in J$. Let $U$ be a preimage of $F_{20}$ modulo $J$ and put $\bar{U}=U /\langle a\rangle, \bar{J}=J /\langle a\rangle$. Then $\bar{J}$ is a minimal normal subgroup of $\bar{U}$. Since $F_{20}=\bar{U} / \bar{J}$ acts faithfully on $\bar{J}$, we have $C_{\bar{U}}(\bar{J})=\bar{J}$. By [10, II.3.3. Satz], $\bar{J}$ has a complement $\bar{H}$ in $\bar{U}, \bar{H} \cong F_{20}$. Let $H$ be the preimage of $\bar{H}$. Then $H$ has a unique (hence normal) 5-Sylow and $H \cong C_{8} \ltimes C_{5}$. This shows $N_{G}(P) \cong C_{8} \ltimes C_{5}$. For the last statement, record that $U \cap J_{0}$ is either 1 or $J_{0}$.

The following proposition will apply in all of our examples of Bol loop folders of exponent 2. We hope that it will also apply in future constructions not considered here. Recall that $\mathrm{O}_{2}(G)$ is the largest normal 2-subgroup of $G$.

Proposition 3.5. Assume $G$ is a finite group, $J=O_{2}(G)$ and $G^{+}=G / J \cong S_{5}$. We denote by $g^{+}$the element of $S_{5}$ corresponding to $g J$. Set $L=G^{\prime} J, K_{1}$ the involutions in $G \backslash L, K_{0}$ a $G$-invariant subset of $J$ containing 1 such that $K_{0} \backslash\{1\}$ consists of involutions, and $H \leq G$. Set $K=K_{0} \cup K_{1}, n_{0}=\left|K_{0}\right|$, and $n_{1}=$ $\left|K_{1} \cap a J\right|$ for $a \in K_{1}$. Assume the following.

(a) $\left(J, H \cap J, K_{0}\right)$ is a Bol loop folder of exponent 2.

(b) $n_{0}=2 n_{1}$.

(c) $\left|G^{+}: H^{+}\right|=6$.

(d) For each $a \in K_{1}, C_{H \cap J}(a)=1$.

(e) Every involution of $L$ is contained in $J$.

Then $(G, H, K)$ is a Bol loop folder of exponent 2 , and $|K|=6 n_{0}=12 n_{1}$.

Proof. First $K_{1}^{+}$is the set of transpositions of $S_{5}$, so $\left|K_{1}^{+}\right|=10$. This implies that $n_{1}$ is well defined. Indeed, for $a, b \in K_{1}, a J, b J$ are conjugate; hence $K_{1} \cap a J, K_{1} \cap b J$ are conjugate in $G$. Moreover, $\left|K_{1}\right|=10 n_{1}$ and by (b),

$$
|K|=\left|K_{0}\right|+\left|K_{1}\right|=2 n_{1}+10 n_{1}=12 n_{1}=6 n_{0} .
$$

Next by (a) and (c),

$$
|G: H|=|G: H J||H J: H|=\left|G^{+}: H^{+}\right||J: J \cap H|=6\left|K_{0}\right|=6 n_{0},
$$

so $|G: H|=|K|$.

We claim $x y \notin H$ for distinct $x, y \in K$. If so, as $|G: H|=|K|, K$ is a set of coset representatives for $H$ in $G$. Then as $K$ is $G$-invariant and $K \backslash\{1\}$ consists of involutions, $(G, H, K)$ is a Bol loop folder of exponent 2 .

If $x, y \in J$, then $x, y \in K_{0}$, so $x y \notin H$ by (a). Next $K_{1}^{+} \cap H^{+}=\emptyset$, so if $x \in J$ and $y \in K_{1}$, then $(x y)^{+}=y^{+} \notin H^{+}$, so $x y \notin H$. Thus we may take $x, y \in K_{1}$ and $x y \in H$. Now as $K_{1}^{+}$is a set of transpositions in $S_{5}$, the order of $(x y)^{+}$is 1,2 
or 3 . Since $H^{+} \cong F_{20}$ has no element of order 3 , we get $(x y)^{2} \in J$. In particular, $D=\langle x, y\rangle$ is a 2-group. Let $z$ be the unique involution in $\langle x y\rangle$. By $x y \in L$ and (e), $z \in H \cap J$. Moreover, $x, y$ commute with $z$, which contradicts (d).

Remark. The fact that (e) is necessary can be seen from the counterexample $G=$ $S_{5} \ltimes J$.

Theorem 3.6. Assume $G$ is a group satisfying condition (*) of Lemma 3.3. Let $J_{0}$ be the minimal normal subgroup of $G$ and put $K=J_{0} \cup c^{G}$. Define $H=$ $N_{G}(P)$ where $P$ is a 5-Sylow subgroup of $G$. Then $(G, H, K)$ is a Bol loop folder determining a simple Bol loop of exponent 2 of order 96 . Conversely, if $\left(G, H^{*}, K^{*}\right)$ is an exponent 2 Bol loop folder, then $H^{*}$ is a conjugate of $H$ and $K^{*}=K$.

Proof. With the notation of Proposition 3.5, $K_{0}=J_{0}$ and $K_{1}=c^{G}$. Then $n_{0}=16$, $n_{1}=\left|c^{G} \cap c J\right|=80 / 10=8$ and $\left|G^{+}: H^{+}\right|=6$, so (b) and (c) hold. Part (e) follows from Lemma 3.4 (ii). Since $J$ is elementary Abelian, $H \cap J$ consists of 1 and the unique involution of $H$. This involution cannot be centralized by $c$; otherwise it would be central in $G=\langle c, P, J\rangle$. Hence (d) follows. Finally, $H \cap J$ is not contained in $J_{0}$; therefore $J_{0}$ is a complement to $H \cap J$ in $J$, showing (a). By Proposition 3.5, $(G, H, K)$ is a Bol loop folder of exponent 2 .

For the converse, we observe that $\left(G, H^{*}, K^{*}\right)$ determines a Bol loop of exponent 2 with all proper subloops solvable. Thus, by the Main Theorem of [3], $H^{*}$ maps surjectively to $F_{20}$. By Lemma 3.4(iii), $H^{*}=H$ or $H^{*}=H J_{0}$ up to conjugation. In the latter case, the loop has order 6, which is impossible. Again by Aschbacher's result, $c^{G} \subset K^{*}$. Finally, if $J_{0} \nsubseteq K^{*}$, then $K^{*}$ will contain a conjugate of the involution of $H$, which is not possible. This proves the theorem.

As the group given in Lemma 3.2 satisfies $(*)$, we have

Corollary 3.7. There exists a simple Bol loop of exponent 2 and order 96.

Remark. The Bol loop folder $(G, H, K)$ of Theorem 3.6 was discovered independently by B. Baumeister and A. Stein [5] (Free University of Berlin), as well.

\section{4. $S_{5}$-MODULES OVER $\mathbb{F}_{2}$}

In this section we collect some useful facts about $k S_{5}$-modules, where $k$ is a field of characteristic 2 .

Lemma 4.1. The group $S_{5}$ has three absolutely irreducible representations over $\mathbb{F}_{2}$ : the trivial representation and two representations $M, N$ of dimension 4 . The two 4-dimensional modules can be distinguished by the fact that $C_{M}(x)=0$ and $\operatorname{dim}_{\mathbb{F}_{2}}\left(C_{N}(x)\right)=2$ for an element $x \in S_{5}$ of order 3 . Moreover, the following hold.

(i) $M$ is the 4-dimensional irreducible component in the 6-dimensional permutation module for $S_{5} \cong P G L(2,5)$. Also, let $V$ be the natural 2-dimensional module of $A_{5} \cong S L(2,4)$ over the field $\mathbb{F}_{4}$ and let $\sigma$ be the semilinear map of $V$ induced by the Frobenius automorphism of $\mathbb{F}_{4}$. Then $S_{5} \cong S L(2,4) \rtimes\langle\sigma\rangle$ and $V$ is a 4-dimensional $S_{5}$-module over $\mathbb{F}_{2}$. The $S_{5}$-modules $M$ and $V$ are isomorphic.

(ii) $N$ is the 4-dimensional irreducible component in the 5-dimensional permutation module of $S_{5}$. Also if $N$ is a 4-dimensional orthogonal space of Witt index 1 over $\mathbb{F}_{2}$, then $O(N)=O_{4}^{-}(2) \cong S_{5}$. Note that $N$ has 5 singular and 10 nonsingular vectors and these are the $S_{5}$-orbits on $N$. 
(iii) $N$ is absolutely irreducible as an $A_{5}$-module. $M$ is irreducible but not absolutely as an $A_{5}$-module, the splitting field being $\mathbb{F}_{4}$. In particular, the modules are nonisomorphic as $A_{5}$-modules.

(iv) $N$ and $M$ are isomorphic absolutely irreducible projective $F_{20}$-modules.

Proof. Let us first define $N, M$ as irreducible components of the permutation modules. By [14, Table 1], they are absolutely irreducible. As $S_{5}$ has 3 classes of elements of odd order, by [1, Theorem 3.2] $S_{5}$ has no other absolutely irreducible modules over $F_{2}$. The properties of $N, M$ can be verified by straightforward calculations; the irreducibility as $A_{5^{-}}$and $F_{20}$-modules follows again from 14, Table 1]. We show $N_{F_{20}} \cong M_{F_{20}}$. As $F_{20}$ has two classes of elements of odd order, $F_{20}$ has two absolutely irreducible modules: the trivial one and $N_{F_{20}}$ coming from the 2-transitive permutation representation. So if $M_{F_{20}}$ were not isomorphic to $N_{F_{20}}$, then it could be brought to upper triangular form over $\overline{\mathbb{F}}_{2}$, which is clearly impossible.

It remains to show that $N_{F_{20}}$ is projective. Since $N_{C_{4}}$ is isomorphic to the group algebra $\mathbb{F}_{2} C_{4}$, it is a projective $C_{4}$-module by [1, Theorem 4.2]. Using [1, Corollary 9.3], we obtain that $N$ is projective as an $F_{20}$-module.

We observe that these $S_{5}$-modules can immediately be constructed using the Steinberg Tensor Product Theorem [18, Theorem 13.1], as well.

We will now construct an $S_{5}$-module $U$ which will play a central role in the generalization of our first construction of a Bol loop of exponent 2.

Let $U=U_{1} \oplus U_{2}$ be the direct sum of two copies of $N$ as an $\mathbb{F}_{2} A_{5}$-module. As $U_{i}$ is an orthogonal space, we can regard $U$ as an orthogonal space which is the orthogonal direct sum of the two nondegenerate subspaces $U_{1}$ and $U_{2}$. The stabilizer $B$ of $\left\{U_{1}, U_{2}\right\}$ in $O(U)$ is $\left(G_{1} \times G_{2}\right)\langle\tau\rangle$ where

$$
G_{i}=C_{O(U)}\left(U_{3-i}\right) \cong O\left(U_{i}\right) \cong S_{5}
$$

and $\tau$ is an involution interchanging $U_{1}, U_{2}$. Thus $B$ is the wreath product of $S_{5}$ with $C_{2}$. In particular, the elements $\tau$ and $\left(g_{1}, g_{2}\right) \in G_{1} G_{2}$ map $u_{1} \oplus u_{2} \in U$ to

$$
\left(u_{1} \oplus u_{2}\right) \tau=u_{2} \oplus u_{1} \text { and }\left(u_{1} \oplus u_{2}\right)\left(g_{1}, g_{2}\right)=u_{1} g_{1} \oplus u_{2} g_{2},
$$

respectively. Set

$$
G_{0}=C_{G_{1} G_{2}}(\tau)=\left\{(g, g) \mid g \in S_{2}\right\} \cong S_{5},
$$

and let $L=\left[G_{0}, G_{0}\right] \cong A_{5}, t_{0}$ an involution (transposition) in $G_{0} \backslash L, t=t_{0} \tau$, and $D=L\langle t\rangle$. Then $t \tau=\tau t$ and the action of $t$ on $U$ is

$$
\left(u_{1} \oplus u_{2}\right) t=u_{2} c \oplus u_{1} c,
$$

where $c \in S_{5}$ is the transposition corresponding to $t_{0}$. It is immediate that $D \cong S_{5}$.

Set $W=C_{U}(\tau)$. Then $W$ is an $\mathbb{F}_{2} D$-submodule of $U$, and also

$$
W=[U, \tau]=\left\{u+u \tau \mid u \in U_{1}\right\}=\{u \oplus u \mid u \in N\},
$$

with the map $u \mapsto[u, \tau]=u+u \tau$ an $\mathbb{F}_{2} L$-isomorphism of $U_{1}$ with $W$. If $Q$ is the quadratic form on $U$, then as $Q\left(u_{1} \oplus u_{2}\right)=Q\left(u_{1}\right)+Q\left(u_{2}\right)$ and $Q(u)=Q(u \tau),[u, \tau]$ is singular, so $W$ is totally singular.

Lemma 4.2. With the notation above, we have the following.

(i) $U$ has 3 irreducible $L$-submodules, namely $U_{1}, U_{2}$ and $W$.

(ii) $W$ is the unique proper $D$-submodule of $U$. 
(iii) Let $P$ be a Sylow 5-subgroup of $D, D_{1}=N_{D}(P)$. Then $D_{1} \cong F_{20}$ and $U$ has precisely $3 D_{1}$-submodules $W, T_{1}, T_{2}$.

(iv) The orbits of $D_{1}$ on $T_{i}$ have length $1,5,10$. In particular, each member of $T_{i}$ is fixed by some involution of $D_{1}$.

Proof. By Lemma 4.1, $N$ is projective as an $F_{20}$-module. Since $U / W$ is a 4 dimensional irreducible for $D_{1}, U_{D_{1}}$ splits over $W_{D_{1}} \cong N_{D_{1}}$ and hence $U=W \oplus T_{1}$ with $D_{1}$-submodule $T_{1}$. Again by Lemma 4.1, $N_{L}$ and $N_{D_{1}}$ are completely irreducible. Schur's lemma then implies $\operatorname{End}_{\mathbb{F}_{2} L}(N)=\operatorname{End}_{\mathbb{F}_{2} D_{1}}(N)=\mathbb{F}_{2}$. We can now apply [2, (27.14)] to obtain (i) and (iii). Part (ii) follows from (i). Finally, (iv) holds since $T_{i}$ and $W$ are $D_{1}$-isomorphic and $W$ is the permutation module modulo the center.

In the next lemma, we keep using the above notation.

Lemma 4.3. (i) $\operatorname{dim} C_{U}(t)=4, C_{U}(t)+T_{1}=U$ and $C_{U}(t) \cap T_{1}=0$.

(ii) Under the action of $A_{5}$ on the submodules $W, U_{1}, U_{2}$ of $U$, the lengths of the orbits are $1,5,10$. Let $S_{0}, S_{1}, S_{2}$ be the orbits of length 5 in $W, U_{1}, U_{2}$, respectively. Then $S=\{0\} \cup S_{0} \cup S_{1} \cup S_{2}$ is a (nonlinear) $S_{5}$-invariant complement to $T_{1}$ in $U$; that is, $S+T_{1}=U$.

Proof. (i) We have $D=L\langle t\rangle \cong S_{5}$. Let us denote the element of $S_{5}$ corresponding to $a \in D$ by $a^{+}$; w.l.o.g. we can assume $t^{+}=(12)$ and $P^{+}=\langle(12345)\rangle$. Then $D_{1}^{+}=\langle(12345),(1325)\rangle$. Let $b \in D$ such that $b^{+}=(12)(35)$. Then $b \in D_{1}$ and $b$ commutes with $t$ and $\tau$. Record that the action of $b, t, \tau$ on $U$ is

$$
\begin{aligned}
& b \quad: \quad u_{1} \oplus u_{2} \mapsto u_{1} b^{+} \oplus u_{2} b^{+}, \\
& \tau \quad: \quad u_{1} \oplus u_{2} \mapsto u_{2} \oplus u_{1}, \\
& t \quad: \quad u_{1} \oplus u_{2} \mapsto u_{2} t^{+} \oplus u_{1} t^{+} .
\end{aligned}
$$

Define the element $\tilde{c} \in B$ by

$$
\tilde{c}: u_{1} \oplus u_{2} \mapsto u_{1} t^{+} \oplus u_{2} .
$$

Then $\tilde{c}$ commutes with $b$ and $\tau^{\tilde{c}}=t$. Put $E_{1}=\langle t, b\rangle$; clearly $E_{1}^{\tilde{c}}=\langle\tau, b\rangle$. On the one hand, we have

$$
\begin{aligned}
\operatorname{dim}_{\mathbb{F}_{2}}\left(C_{U}\left(E_{1}\right)\right) & =\operatorname{dim}\left(C_{U}(\tau, b)\right) \\
& =\operatorname{dim} C_{C_{U}(b)}(\tau) \\
& =\operatorname{dim} C_{C_{U_{1}}(b) \oplus C_{U_{2}}(b)}(\tau) \\
& =\operatorname{dim} C_{U_{1}}(b) \\
& =\operatorname{dim} C_{N}\left(b^{+}\right)=2 .
\end{aligned}
$$

We show on the other hand that $\operatorname{dim}_{\mathbb{F}_{2}}\left(C_{W}\left(E_{1}\right)\right) \geq 2$. Indeed, the $S_{5}$-modules $W$ and $N$ are isomorphic and $\operatorname{dim}\left(C_{W}(t)\right)=\operatorname{dim}\left(C_{N}\left(t^{+}\right)\right)=3$. Then $C_{W}\left(E_{1}\right)=$ $C_{C_{W}(t)}(b)$ is of rank at least $\operatorname{dim}\left(C_{W}(t)\right) / 2=3 / 2$.

Now, from $\operatorname{dim}\left(C_{W}\left(E_{1}\right)\right) \geq 2$ it follows that $C_{U}\left(E_{1}\right) \leq W$. However if $C_{T_{1}}(t) \neq$ 0 , then $C_{T_{1}}\left(E_{1}\right) \neq 0$, contradicting $T_{1} \cap W=0$ and $C_{U}\left(E_{1}\right) \leq W$.

(ii) We have seen that $S_{i}$ is the set of singular points in $U_{i}$ for $i=1,2$. The action of $D_{1} \cong F_{20}$ on $S_{i}$ is its natural 2-transitive action on 5 points. Here $D_{1} \cap L$ contains precisely 5 involutions and each member of $S_{i}$ is fixed by exactly one involution of $D_{1} \cap L$. Moreover, each member of $T_{1}$ is fixed by some involution of $D_{1}$. 
We have to show that for distinct $x, y \in S, x+y \notin T_{1}$; then $S$ is a complement to $T_{1}$ by an order argument. Assume $x+y \in T_{1}$ and denote by $a$ an involution of $D_{1}$ fixing $x+y$. Then $x, y$ are the projections of $x+y$ on $S_{i}, S_{j}$, so $a$ fixes the projections $x$ and $y$. As $T_{1} \cap U_{i}=0$ for $0 \leq i \leq 2, x \in S_{i}$ and $y \in S_{j}$ for some $i \neq j$. If $x \in S_{1}$ and $y \in S_{2}$, then $x$ and $y$ are the unique fixed points of $a$ in $S_{1}, S_{2}$. By $a \tau=\tau a, x \tau=y$ holds, and hence $x+y=[x, \tau] \in W$, contradicting $T_{1} \cap W=0$.

Thus we may take $x \in S_{0}$ and $y \in S_{1}$. Then $x=x_{1}+x_{2}$ with $x_{i}=S_{i}$, and as $x \in S_{0}, x_{2}=x_{1} \tau$ with $x_{i} \in S_{i}$. Now, $x_{i}$ is the unique fixed point of $a$ in $S_{i}$ and $y$ the unique fixed point of $a$ in $S_{1}$, so $y=x_{1}$ and $x+y=x_{1}+x_{2}+y=x_{2} \in T_{1} \cap U_{2}=0$, a contradiction.

\section{An infinite family of Simple Bol LOOPS OF EXPONENT 2}

In this section, $G$ denotes a group satisfying condition (*) of Lemma $3.3 \mathrm{H}$ is the normalizer of a 5-Sylow $P$ of $G$, and $c$ is an involution from $G \backslash[G, G] J$. The $S_{5}$-modules $N$ and $U$ are defined as in Section 4, Also $U=U_{1} \oplus U_{2}=T_{1} \oplus T_{2}$ where $U_{1}, U_{2}$ are $A_{5}$-submodules and $T_{1}, T_{2}$ are $F_{20}$-submodules. Moreover, $U_{1}, U_{2}, T_{1}, T_{2}$ are different from the unique $S_{5}$-submodule $W$ of $U$. All these subspaces are irreducible $\mathbb{F}_{2} P$-modules, which implies $U_{i} \cap T_{j}=0$.

Let us fix a positive integer $k$ and put

$$
\mathscr{U}=U^{k}, \mathscr{U}_{i}=U_{i}^{k}, \mathscr{T}_{i}=T_{i}^{k}, \mathscr{W}=W^{k} .
$$

Clearly, $\mathscr{W}$ is an $S_{5}$-submodule and $\mathscr{U}=\mathscr{U}_{1} \oplus \mathscr{U}_{2}=\mathscr{T}_{1} \oplus \mathscr{T}_{2}$. We write $\mathscr{G}=G \ltimes \mathscr{U}$ where $J \triangleleft G$ acts trivially on the $S_{5}$-module $\mathscr{U}$. Moreover,

$$
\mathscr{J}=O_{2}(\mathscr{G})=\langle J, \mathscr{U}\rangle \text {. }
$$

We will consider the elementary Abelian subgroup $\mathscr{J}$ of $\mathscr{G}$ as an $S_{5}$-module over the field $\mathbb{F}_{2}$. In particular, with some abuse of notation, we will denote the group operation on $\mathscr{J}$ additively and write $\mathscr{J}=J+\mathscr{U}$, etc. It is easy to see that

$$
\mathscr{G} / \mathscr{J} \cong S_{5} \text { and } \operatorname{soc}(\mathscr{G})=J_{0}+\mathscr{W} \text {. }
$$

Moreover, since $J_{0}$ and $W$ are nonisomorphic $S_{5}$-modules, $J_{0} \oplus W$ does not contain diagonal submodules. This implies that for any minimal submodule $M$ of $\mathscr{J}$, we either have $M=J_{0}$ or $M \leq \mathscr{W}$.

The action of the involution $c \in G \backslash J$ on $\mathscr{U}$ equals the action of the transposition (12) $\in S_{5}$; hence $c$ interchanges $\mathscr{U}_{1}, \mathscr{U}_{2}$. This implies $\left|C_{\mathscr{U}}(c)\right|=16^{k}$ and $\left|c^{\mathscr{U}}\right|=16^{k}$; that is, $\mathscr{U}$ is transitive on the involutions in $c \mathscr{U}$. As $G \cong \mathscr{G} / \mathscr{U}$ is transitive on the 80 involutions in $G \backslash J$ and as $\mathscr{U}$ is transitive on the $16^{k}$ involutions on $c \mathscr{U}, \mathscr{G}$ is transitive on the $80 \cdot 16^{k}$ involutions in $\mathscr{G} \backslash \mathscr{J}$.

Using Aschbacher's Main Theorem [3], we conclude that a Bol loop folder $(\mathscr{G}, \mathscr{H}, \mathscr{K})$ must have the following properties: The index of $\mathscr{H}$ has to be $96 \cdot 16^{k}$; that is, $\mathscr{H}$ must have order $40 \cdot 16^{k}$. The set of involutions $\mathscr{K}$ is the union of $c^{\mathscr{G}}$ and $\mathscr{K} \cap \mathscr{J}$; thus, $|\mathscr{K} \cap \mathscr{J}|=16 \cdot 16^{k}$.

There are very many possible choices for $\mathscr{H}$ and $\mathscr{K}$. The most obvious choice is the following.

Proposition 5.1. Put $\mathscr{H}=H \ltimes \mathscr{T}_{1}$ and $\mathscr{K}=c^{\mathscr{G}} \cup\left(J_{0} \oplus \mathscr{W}\right)$. Then $(\mathscr{G}, \mathscr{H}, \mathscr{K})$ is a Bol loop folder. Moreover, the homomorphism $\mathscr{G} \rightarrow G$ with kernel $\mathscr{U}$ induces a surjective homomorphism between the loop folders $(\mathscr{G}, \mathscr{H}, \mathscr{K})$ and $(G, H, K)$. In other words, the Bol loop corresponding to $(\mathscr{G}, \mathscr{H}, \mathscr{K})$ is an extension of the elementary Abelian group of order $2^{2 k}$ by the loop corresponding to $(G, H, K)$. 
Proof. We apply Proposition 3.5. (a), (c) and (e) are trivial. Part (b) follows from

$$
n_{1}=\left|c^{\mathscr{G}} \cap c \mathscr{J}\right|=\left|C_{\mathscr{J}}(c)\right|=8\left|C_{\mathscr{U}}(c)\right|=8 \cdot 16^{k}
$$

and $n_{0}=\left|J_{0}\right||\mathscr{W}|=16 \cdot 16^{k}=2 n_{1}$. For (d), we use

$$
C_{\mathscr{H} \cap \mathscr{J}}(c)=C_{H \cap J+\mathscr{T}_{1}}(c)=C_{H \cap J}(c)=1,
$$

as by Lemma 4.3, $\mathscr{T}_{1} \cap \mathscr{T}_{1}^{c}=0$.

In the rest of this section, for each integer $k \geq 1$, we modify these $\mathscr{H}$ and $\mathscr{K}$ such that the resulting loop will be simple. Let $U^{*}$ be a copy of $U$ in $\mathscr{U}$ such that $\mathscr{U}=U^{*} \oplus U^{k-1}$. We denote the subspaces corresponding to $T_{i}, U_{i}, W$ by $T_{i}^{*}, U_{i}^{*}, W^{*}$. Let us define the set $S \subseteq U$ as in Lemma 4.3(ii) and let $S^{*}$ be the corresponding subset of $U^{*}$. In order to construct the new $\mathscr{K}$, we simply replace $W^{*}$ by $S^{*}$.

Let $\psi: J_{0} \rightarrow T_{1}^{*}$ be an isomorphism of $F_{20}$-modules and define

$$
\mathscr{T}_{\psi}=\left\{v+\psi(v)+u \mid v \in J_{0}, u \in T_{1}^{k-1}\right\} .
$$

Then $\mathscr{T}_{\psi}$ is normalized by $H$ and we define the new subgroup $\mathscr{H}$ of $\mathscr{G}$ by $\mathscr{H}=$ $H \ltimes \mathscr{T}_{\psi}$.

Theorem 5.2. Let $\mathscr{H}=H \ltimes \mathscr{T}_{\psi}, \widetilde{\mathscr{W}}=\left(\mathscr{W} \backslash W^{*}\right) \cup S^{*}$ and $\mathscr{K}=c^{\mathscr{G}} \cup\left(J_{0}+\widetilde{\mathscr{W}}\right)$. Then the triple $(\mathscr{G}, \mathscr{H}, \mathscr{K})$ is a Bol loop folder such that the corresponding Bol loop is simple of exponent 2.

Proof. Again, the first statement follows from Proposition 3.5 and Lemma 4.3, one needs to verify hypothesis (a) and (d) of the proposition only. Again (d) follows from $T_{1} \cap T_{1}^{c}=0$.

We prove (a) by showing that $\mathscr{K}_{0}=\mathscr{K} \cap \mathscr{H} \mathscr{J}$ is a transversal to $\mathscr{H}$ in $\mathscr{H} \mathscr{J}$. Since $\mathscr{K}_{0} \subset \mathscr{J}$, this is equivalent to the fact that $\mathscr{K}_{0}=J_{0}+\widetilde{\mathscr{W}}$ is a complement of the subspace

$$
\mathscr{H} \cap \mathscr{J}=H \cap J+\mathscr{T}_{\psi}
$$

in $\mathscr{J}$, i.e.,

$$
\mathscr{K}_{0}+\mathscr{H} \cap \mathscr{J}=\mathscr{J} .
$$

In order to show this, we use the identities

$$
\begin{aligned}
\mathscr{W}+\mathscr{T}_{1} & =\mathscr{U}, \\
J_{0}+\mathscr{T}_{\psi} & =J_{0}+\mathscr{T}_{1}, \\
J_{0}+\mathscr{H} \cap \mathscr{J} & =J_{0}+H \cap J+\mathscr{T}_{\psi} \\
& =J_{0}+H \cap J+\mathscr{T}_{1} .
\end{aligned}
$$

Then

$$
\begin{aligned}
J_{0}+\mathscr{W}+\mathscr{H} \cap \mathscr{J} & =J_{0}+\mathscr{W}+H \cap J+\mathscr{T}_{\psi} \\
& =J_{0}+\mathscr{W}+H \cap J+\mathscr{T}_{1} \\
& =J_{0}+\mathscr{U}+H \cap J \\
& =J+\mathscr{U} \\
& =\mathscr{J} ;
\end{aligned}
$$

that is, $J_{0}+\mathscr{W}$ is a complement to $\mathscr{H} \cap \mathscr{J}$ in $\mathscr{J}$ by the order argument

$$
\left|J_{0}+\mathscr{W}\right||\mathscr{H} \cap \mathscr{J}|=|\mathscr{J}| .
$$


We constructed $\widetilde{\mathscr{W}}$ from $\mathscr{W}$ by deleting the minimal submodule $W^{*}$ and replacing it by $S^{*}$. Therefore, it is enough to show that this deformation of $\mathscr{W}$ does not change the property of being a complement:

$$
\begin{aligned}
J_{0}+W^{*}+\mathscr{H} \cap \mathscr{J} & =J_{0}+W^{*}+H \cap J+\mathscr{T}_{\psi} \\
& =H \cap J+J_{0}+W^{*}+\mathscr{T}_{1} \\
& =H \cap J+J_{0}+W^{*}+T_{1}^{*}+\mathscr{T}_{1} \\
& =H \cap J+J_{0}+S^{*}+T_{1}^{*}+\mathscr{T}_{1} \\
& =J_{0}+S^{*}+H \cap J+\mathscr{T}_{\psi} \\
& =J_{0}+S^{*}+\mathscr{H} \cap \mathscr{J} .
\end{aligned}
$$

This proves (a); hence $(\mathscr{G}, \mathscr{H}, \mathscr{K})$ is a Bol loop folder.

It remains to show that the Bol loop $\mathscr{Q}$ corresponding to $(\mathscr{G}, \mathscr{H}, \mathscr{K})$ is simple. Let us therefore assume that $\mathscr{Q} \rightarrow \mathscr{Q}^{\sharp}$ is a nontrivial surjective loop homomorphism and let $\left(\mathscr{G}^{\sharp}, \mathscr{H}^{\sharp}, \mathscr{K}^{\sharp}\right)$ be the loop folder of $\mathscr{Q}^{\sharp}$. Then we have a surjective homomorphism $\alpha: \mathscr{G} \rightarrow \mathscr{G}^{\sharp}$ with $\alpha(\mathscr{H})=\mathscr{H}^{\sharp}$ and $\alpha(\mathscr{K})=\mathscr{K}^{\sharp}$. Let $\mathscr{N}=\operatorname{ker} \alpha$ and $c^{\sharp}=\alpha(c)=c \mathscr{N}$. On the one hand, $\mathscr{H}^{\sharp}$ is core-free; thus,

$$
\operatorname{core}_{\mathscr{G}}(\mathscr{H} \mathscr{N})=\mathscr{N} \text {. }
$$

On the other hand, $\mathscr{N} \leq \mathscr{J}$ since otherwise $\mathscr{H} \mathscr{N}=\mathscr{G}$ and $\mathscr{Q}^{\sharp}=1$.

Let us first assume that $J_{0} \leq \mathscr{N}$. Since

$$
J / J_{0} \leq Z\left(\mathscr{G} / J_{0}\right) \triangleleft \mathscr{G} / J_{0} \text { and } J / J_{0} \leq \mathscr{H} \mathscr{N} / J_{0},
$$

we have $J \leq \mathscr{N}$ by (2). In this case the image $G^{\sharp}$ of $G \leq \mathscr{G}$ is a homomorphic image of $G / J \cong S_{5}$. Furthermore, if $\left[c^{\sharp}, c^{\sharp g}\right]=1$, then $c^{\sharp} c^{\sharp g}$ normalizes a Sylow 5 -subgroup of $G^{\sharp}$; thus, $c^{\sharp} c^{\sharp g}$ is contained in a conjugate of $\mathscr{H}^{\sharp}$, and hence $c^{\sharp}=c^{\sharp g}$ in this case. As the commuting graph of transpositions in $S_{5}$ is connected, $c^{\sharp}=c^{\sharp}$ for all $g$. This means $[c, \mathscr{G}] \leq \mathscr{N}$, contradicting $\mathscr{N} \leq \mathscr{J}$.

Let us now assume $J_{0} \not \leq \mathscr{M}$ and let $M$ be a minimal normal subgroup of $\mathscr{G}$ contained in $\mathscr{N}$. Then $M \leq \operatorname{soc}(\mathscr{G})=J_{0}+\mathscr{W}$. Since $J_{0}$ and $W$ are nonisomorphic $S_{5}$-modules, $J_{0}+\mathscr{W}$ contains no submodules isomorphic to $J_{0}$ and different from $J_{0}$. This implies $M \leq \mathscr{W}$ and, in particular, $\mathscr{N} \cap \mathscr{W} \neq 0$.

Let us take an element $s \in S^{*} \backslash W^{*} \subseteq \mathscr{K}$. As $W^{*}$ and $T_{0}^{*}$ are complements in $U^{*}, s \neq 0$ has the unique decomposition $s=w+t$ with $0 \neq w \in W^{*}$ and $0 \neq t \in T_{1}^{*}$. Furthermore, for $0 \neq j=\psi^{-1}(t) \in J_{0}, j+t \in \mathscr{T}_{\psi} \leq \mathscr{H}$ holds. We claim that $j+t \in \mathscr{N}$. Indeed, we have the decomposition

$$
w=(s+j)+(j+t), \quad s+j \in \mathscr{K}, j+t \in \mathscr{H} .
$$

If $\mathscr{N} \leq U^{*}$, then $M=W^{*}$ and $\alpha(s+j)=\alpha(j+t) \in \mathscr{K}^{\sharp} \cap \mathscr{H}^{\sharp}=1$. In particular, $j+t \in \mathscr{N}$. If $\mathscr{N} \not U^{*}$, then for an arbitrary element $n \in(\mathscr{N} \cap \mathscr{W}) \backslash U^{*}, w+n \in \mathscr{K}$. This means that the element $\alpha(w)$ has two $\mathscr{H}^{\sharp} \mathscr{K}^{\sharp}$ decompositions:

$$
\alpha(w)=\alpha(w+n)+0=\alpha(s+j)+\alpha(j+t) .
$$

This is only possible if $j+t \in \mathscr{N}$; thus our claim is proved.

Let $M^{\prime}$ be the $S_{5}$-submodule generated by $j+t$. Then $J_{0} \leq M^{\prime} \leq \mathscr{N}$ as the irreducible $J_{0}$ is not $S_{5}$-isomorphic to a submodule of $U^{*}$. This contradiction proves the simplicity of $\mathscr{Q}$. 
Remark. We have seen that there are at least two possibilities for the choice of $\mathscr{H}$. Also in $\widetilde{\mathscr{W}}$, we can replace any minimal submodule $W^{* *}$ by an appropriate $S^{* *}$. This shows that there are many Bol loops of exponent 2 which live in the same nonsolvable group. Many of these loops are simple. Using computer calculations, we were able to construct over 30 nonisomorphic simple Bol loops of exponent 2 in $\mathscr{G}$ in the case $k=1$.

In fact, this phenomena in not unusual for Bol loops of exponent 2. In 12 , Section 5] and [16. Theorem 5.5], the authors constructed rich classes of Bol loops of exponent 2 having the same enveloping groups, namely the wreath product $C_{2}^{n}$ ? $C_{2}$ and the extra special 2-group $E_{2^{2 n+1}}^{+}$, respectively. In these cases, a simple parametrization of the conjugacy classes of involutions enabled a description of the associated loops. Unfortunately, the group $\mathscr{G}$ has many conjugacy classes of involutions and these classes have no nice algebraic parametrization. Therefore, we see no way of classifying all simple Bol loops with enveloping group $\mathscr{G}$.

The above remark lets us make another observation. While the class of finite Bol loops of exponent 2 is very rich, the structure of the right multiplication group of a Bol loop of exponent 2 is rather restricted. In other words, while the classification of finite simple Bol loops of exponent 2 seems to be hopeless, we think that the classification of right multiplication groups of such loops could be a meaningful project.

We finish this paper with the following

Problem. Classify those almost simple groups $T$ for which an exponent 2 Bol loop folder $(G, H, K)$ exists such that $T \cong G / O_{2}(G)$.

\section{ACKNOWLEDGEMENTS}

The author would like to thank Michael Aschbacher (California Institute of Technology) for his detailed comments on the previous version of this paper. He helped to fill some gaps, to simplify the proofs and to replace computer based calculations with theoretical arguments. The author also would like to thank Peter Müller (Würzburg) for many stimulating conversations and helpful comments.

\section{REFERENCES}

[1] J. L. Alperin. Local representation theory. Cambridge University Press, New York, 1993. MR860771 (87i:20002)

[2] M. Aschbacher. Finite group theory. Cambridge Studies in Advanced Mathematics, 10. Cambridge University Press, Cambridge, 1986. MR895134 (89b:20001)

[3] M. Aschbacher. On Bol loops of exponent 2. J. Algebra 288, No. 1, 99-136 (2005). MR2138373 (2006k:20131)

[4] M. Aschbacher, M. K. Kinyon and J. D. Phillips. Finite Bruck loops. Trans. Am. Math. Soc. 358, No. 7, 3061-3075 (2005). MR2216258 (2006m:20111)

[5] B. Baumeister and A. Stein. Private communication (2007).

[6] R. H. BRUCK. A survey of binary systems. Springer-Verlag, Berlin, 1958. MR0093552(20:76)

[7] R. P. Burn. Finite Bol loops I. Math. Proc. Cambridge Philos. Soc. 84(3) (1978), 53-66. MR0492030 (58:11194)

[8] The GAP Group, GAP - Groups, Algorithms, and Programming, Version 4.4.9; 2006. http://www.gap-system.org.

[9] S. Heiss. Self-invariant 1-factorizations of complete graphs, submitted for publication.

[10] B. Huppert. Endliche Gruppen I, Springer-Verlag, Berlin, Heidelberg, 1967. MR0224703 (37:302) 
[11] H. Kiechle. Theory of K-loops. Lecture Notes in Mathematics, 1778. Springer, Berlin, 2002. MR.1899153 (2003d:20109)

[12] H. Kiechle and G. P. Nagy. On the extension of involutorial Bol loops. Abh. Math. Semin. Univ. Hamb. 72, 235-250 (2002). MR1941556(2003m:20095)

[13] E. Kolb and A. Kreuzer. Geometry of kinematic K-loops. Abh. Math. Sem. Univ. Hamburg 65 (1995), 189-197. MR.1359127 (96k:20150)

[14] B. Mortimer. The modular permutation representations of the known doubly transitive groups. Proc. London Math. Soc. (3) 41 (1980), 1-20. MR.579714 (81f:20004)

[15] G. P. NAGY. Solvability of universal Bol 2-loops. Comm. Algebra, 26(2) (1998), 549-555. MR:1604103 (98k:20112)

[16] G. P. Nagy. On the structure and number of small Frattini Bol 2-loops. Math. Proc. Camb. Philos. Soc. 141, No. 3, 409-419 (2006). MR2281406 (2008a:20102)

[17] G. P. Nagy. A class of simple proper Bol loops. Manuscripta Math. 127, No. 1, 81-88 (2008). MR2429915

[18] R. Steinberg. Endomorphisms of linear algebraic groups. Memoirs of the American Mathematical Society, No. 80, American Mathematical Society, Providence, R.I. 1968. MR.0230728 $(37: 6288)$

Bolyai Institute, University of Szeged, Aradi vértanúk tere 1, H-6720 Szeged, HunGARY

E-mail address: nagyg@math.u-szeged.hu

Current address: Mathematisches Institut, Universität Würzburg, Am Hubland, D-97070 Würzburg, Germany 